\title{
Assessing the Nutritional Knowledge, Disease Perception, and Adherence to Diet in Patients with Hypertension Admitted to Farshchian Cardiovascular Clinic in 2017
}

\author{
Masoud Khodaveisi ${ }^{1}$, Shahla Fakhrazizi ${ }^{1}$, Nahid Mohammadi ${ }^{1} \&$ Leili Tapak $^{2}$ \\ ${ }^{1}$ Departmant of Community Health Nursing, School of Nursing and Midwifery, Hamedan University of Medical \\ sciences, Hamedan, Iran \\ ${ }^{2}$ Departmant of Epidemiology, School of Public Health, Hamedan University of Medical Sciences, Hamedan, Iran \\ Correspondence: Leili Tapak, Departmant of Epidemiology, School of Public Health, Hamedan University of \\ Medical Sciences, Hamedan, Iran. E-mail: 1.tapak06@gmail.com
}

Received: June 5, 2017

Accepted: June 25, 2018

Online Published: July 19, 2018

doi:10.5539/jmbr.v8n1p68

URL: https://doi.org/10.5539/jmbr.v8n1p68

\begin{abstract}
Background and objective: adherence to diet by patients to control the blood pressure has always been challenging. The current study was conducted to evaluate the nutritional knowledge status and disease perception and adherence to diet in patients with hypertension.

Methodology: In this quasi-experimental study conducted in 2017, 100 patients with hypertension admitted to Farshchian Cardiovascular Clinic were selected using convenient sampling method and by considering the inclusion criteria. The data were collected using demographic questionnaire, observing nutritional knowledge questionnaire, disease perception questionnaire, and adherence to diet questionnaire in patients with hypertension. Data were analyzed using spss 16 software and descriptive statistics.

Results: based on the results, mean score of knowledge was $4.73 \pm 1.55$. In this study $58 \%$ of the patients did not have knowledge on high fat diet, $56 \%$ of subjects did not have knowledge on red meat diet and $63 \%$ did not have knowledge on confection. The mean score of perception was $29.58 \pm 3.94$, which $38 \%$ of the patients believed that their diseases cannot be controlled with behavior and 43\% were completely opposed that that their disease can lead into early death, and $56 \%$ opposed to restricting the diet. The mean score of follow-up of diet is $9.45 \pm 2.27$. In this study, $46 \%$ of patients did not adhere to their diet at home and $51 \%$ of patients did not adhere to their diet at workplace.

Conclusion: Due to the lack of knowledge and perception of the disease and the effective follow-up of the diet, it is recommended that necessary measures to be taken to prevent complications of hypertension.
\end{abstract}

Keywords: hypertension, nutritional knowledge, nutritional understanding, nutritional follow-up

\section{Introduction}

Coronary artery disease is the leading cause of mortality and disability in industrialized countries and it accounts for $30 \%$ of deaths. Hypertension is one of the most common, chronic, recurrent diseases, leading to many diseases. Hypertension accounts for $5 \%$ of the diseases in world, so that it affects more than a quarter of adult population in world. It is predicted that its rate to increase by $24 \%$ in developed countries and $80 \%$ in developing countries by 2025 (Mills et al., 2016). This disease is very important due to its high prevalence, but what increases its importance is that it is an uncontrolled disease. Studies have revealed that the role of diet and nutrition in controlling this disease is undeniable, but unfortunately, there is much contradictory evidence, and most patients do not actually pay attention to diet instructions, and only less than half of the patients adopt correct diet as part of their treatment. The control of hypertension is important to minimize the likelihood of development of complications caused by hypertension (Hosseini et al., 2014; Ndumele, Shaykevich, Williams, \& Hicks, 2010). It seems that these wrong behaviors and beliefs to be rooted in inaccurate information and poor knowledge of patients with regard to the nature of the disease and diet and nutrition, as one of the reasons for lack of control of hypertension has been reported to be poor nutrition and diet knowledge of the patients and the lack of information in these patients has been considered as an important issue (Sanne, Muntner, Kawasaki, Hyre, \& DeSalvo, 2008; 
Sheahan \& Fields, 2008). While it has been found that knowledge without other factors may not be able to modify the behavior, it can be the determining factor in nutritional behavior of the person (Mohebi et al., 2013). In fact, knowledge of patients is an important factor in the acceptance of treatment and control of these diseases. A wide range of healthcare staff provide their services in the health system, but nurses are the main elements of the system providing these services and play major role in coordinating and providing health services. As nurses work with families in various environments such as clinics and schools, the important role community-based nurse is reducing the health risks, enhancing the health of various populations, and providing service to families (Linderholm \& Friedrichsen, 2010). Thus, given the importance and impact of changes in nutritional behavior and knowledge on enhancing the health and controlling the disease applicability of care, the present study was conducted to evaluate the nutritional knowledge, disease perception, and adherence to diet in patients with hypertension in those admitted to Farshchian Cardiovascular Clinic of in 2017.

\section{Methodology}

This quasi-experimental study was conducted in Farshchian Cardiovascular Clinic in 2017. In this study, 100 patients with hypertension who met the inclusion criteria were selected using the convenient sampling method. Sample size was calculated based on Kamran et al. (2011) study and using the following formula:

$$
\mathrm{N}=\frac{Z^{2 S^{2}}}{2^{2}}=\frac{1.96^{2} \times 10^{2}}{2^{2}}=96
$$

The inclusion criteria of study included age between 30 and 60 years, mild to moderate hypertension approved by physician, having at least one year of history of hypertension, and not having a known underlying severe disease in severe phase (such as metastatic cancer and renal failure).

The data collection tool included a four-part questionnaire consisting of: 1- Demographic characteristics questions. 2- Nutritional knowledge questions: including 10 correct and incorrect questions, score 1 belongs to correct answer and the zero score belongs to wrong answer or I do not know answer. 3- Questions related to hypertension disease perception include 14 questions in a 4-point Likert scale (strongly agree, agree, disagree, and strongly disagree), which scores 1 and 4 belong to them and the range of scores is 14-56. 4- Questions related to diet follow-up: it includes 5 questions based on the 5-point Likert scale (always, often, sometimes, rarely, never), with scores from 1 to 5 are assigned for them and the range of scores is 5-25. To obtain score of each dimension, the scores related to each of the questions of that dimension are aggregated, which the score of questions related to disease perception is 14-56, the score of questions related to diet follow-up is 5-25 and the score of questions related to diet knowledge is $0-10$. Then, to rank the scores, the total scores of each dimension it is calculated based on 100 scores, and the mean scores are ranked in three levels of desirable (mean score above 75), moderate (mean score of 50-75) and undesirable (mean score less than 50). In the study conducted by Kamran et al. (2013), the reliability was examined using internal consistency (Cronbach's alpha), split-half, and Kuder-Richardson methods and validity of the questionnaire was examined using content and construct validity.

The results of Spearman Brown's, Cronbach's, Gutman's, and Kuder-Richardson coefficients in the questionnaire were desirable and showed satisfactory reliability of this questionnaire. The questions of the questionnaire showed high correlation with the total score and the examination of the internal consistency of the questions showed that the questionnaire's questions had acceptable reliability. In factor analysis, all of the questionnaire's questions have acceptable factor load and based on factor analysis, all questionnaire's questions are important and have appropriate construct validity. This questionnaire was also approved by the professors with a validity of above $90 \%$. Cronbach's Alpha for all dimensions was above 80\% (Kamran, Sharifirad, \& Mohebi, 2013). After determining the number of participants admitted to Farshchian Cardiovascular Clinic, those who met the inclusion criteria of study were selected and the researcher explained the research process completely after obtaining their consent. In order to prevent the effect of some confounding variables, their effects are controlled in the data analysis by modeling their effects. Then, the questionnaires were given to both groups. First, a demographic questionnaire was completed, and then, the questionnaire related to nutritional knowledge, disease perceptions, and diet follow-up in patients with hypertension was completed. Data were analyzed by SPSS 16 software and descriptive statistics.

\section{Results}

In this study, a total of 100 patients participated which their demographic characteristics are presented in Table. Based on the results, mean score of knowledge was $4.73 \pm 1.55$. In this study, $58 \%$ of the patients did not have knowledge on high fat diet, $56 \%$ of subjects did not have knowledge on red meat diet and $63 \%$ did not have knowledge on confection. The mean score of perception was $29.58 \pm 3.94$, which $38 \%$ of the patients believed that their diseases cannot be controlled with behavior and $43 \%$ were completely opposed that that their disease can 
lead into early death, and $56 \%$ opposed to restricting the consumption of salt and fat, and $12 \%$ of people did not believe that this disease can be threat for their health. The mean of the scores according to the results of the diet follow-up questionnaire is $9.45 \pm 2.27$. In this study, 46 percent of patients stated they never adhere to proper diet when they are at home, while only 2 percent of patients with hypertension completely adhere to their die at home. Moreover, $29 \%$ of patients stated that they did not adhere to their diet when they are in community and out of the home. Based on these findings, nutritional knowledge, disease perception and nutritional follow-up are among patients with hypertension and follow-up and corrective actions are required.

Table 1. Frequency of demographic variables in patients with hypertension

\begin{tabular}{llll}
\hline demographic variables & f & \% \\
\hline \multirow{2}{*}{ gender } & male & 45 & 45 \\
& female & 55 & 55 \\
job & employed & 28 & 28 \\
& housewife & 72 & 72 \\
& Illiterate & 8 & 8 \\
education & Elementary and secondary & 33 & 33 \\
& High school & 35 & 35 \\
\multirow{3}{*}{ total } & academic & 24 & 24 \\
& & 100 & 100 \\
\hline
\end{tabular}

Table 2. Questions and percentage of responses to nutritional knowledge in patients with hypertension

\begin{tabular}{llc}
\hline & correct & incorrect \\
\cline { 2 - 3 } Phrases related to knowledge & F (\%) & F (\%) \\
\hline Animal fats, such as the natural butter, increase blood pressure & 42 & 58 \\
The consumption of bread and cereal grains reduces blood pressure & 40 & 60 \\
A person with hypertension should not eat oily seeds, such as almonds, pistachios, hazelnuts and walnuts. & 55 & 45 \\
A patient with hypertension needs to eat at least 4 to 5 units of vegetables per day (each unit equals one glass of & 24 & 76 \\
green leafy vegetables or half of a glass of cooked vegetable) & 62 \\
The consumption of milk and other low-fat dairy products is harmful to a person with hypertension & 38 \\
The consumption of red meat is associated with hypertension & 44 & 56 \\
In hypertension, diet is restricted only in terms of salt intake & 67 & 33 \\
Consumption of foods including chicken and fish is involved in hypertension & 65 & 35 \\
A patient with hypertension should avoid using confection & 37 & 63 \\
Sweet drinks such as carbonated soda are harmful for patient with hypertension & 37 & 63 \\
\hline
\end{tabular}

Table 3. Questions and percentage of responses related perception of patients with hypertension

\begin{tabular}{|c|c|c|c|c|}
\hline Questions related to perception & $\begin{array}{l}\text { Completely } \\
\text { disagree } \\
\text { F (\%) } \\
\end{array}$ & $\begin{array}{l}\text { disagree } \\
\text { F (\%) }\end{array}$ & $\begin{array}{l}\text { agree } \\
\text { F (\%) }\end{array}$ & $\begin{array}{l}\text { Completely } \\
\text { agree } \\
\text { F (\%) } \\
\end{array}$ \\
\hline $\begin{array}{l}\text { I believe it is a disease that is not influenced by my behaviors } \\
\text { (uncontrollable with my behavior change) }\end{array}$ & 38 & 36 & 18 & 8 \\
\hline A disease that sometimes gets better, sometimes gets worse & 35 & 40 & 14 & 11 \\
\hline Sometimes it has no symptom & 51 & 33 & 8 & 8 \\
\hline It can be treated with drug or other therapeutic measures & 46 & 29 & 22 & 14 \\
\hline It can be asymptomatic & 48 & 29 & 8 & 15 \\
\hline It might lead to early death & 43 & 36 & 9 & 12 \\
\hline Drugs act better when they have symptom & 43 & 30 & 16 & 11 \\
\hline It can be worsen with my life style and behaviours & 42 & 30 & 14 & 14 \\
\hline It is a treat to my health & 49 & 30 & 9 & 12 \\
\hline It can treated and improved & 42 & 28 & 9 & 21 \\
\hline It needs treatment even if I feel healthy & 49 & 30 & 8 & 13 \\
\hline It can be improved with drug in long term & 57 & 20 & 7 & 16 \\
\hline The treatment plan (drug and nutrition) should be followed-up for lifetime & 59 & 24 & 7 & 10 \\
\hline It requires restriction in intake of salt and fat & 56 & 22 & 12 & 10 \\
\hline
\end{tabular}


Table 4. Questions and percentage responses related to adherence to diet among patients with hypertension

\begin{tabular}{llllll}
\hline Follow-up phrases & never & rarely & sometimes & often & always \\
& $\mathbf{F ( \% )}$ & $\mathrm{F}(\%)$ & $\mathrm{F}(\%)$ & $\mathrm{F}(\%)$ & $\mathrm{F}(\%)$ \\
\hline When I am at home, I follow the recommended diet (low salt) & 46 & 41 & 6 & 5 & 2 \\
When I am at work, I follow and adhere to diet & 51 & 35 & 6 & 4 & 4 \\
I adhere to diet when I am doing exercise & 43 & 39 & 11 & 5 & 2 \\
I adhere to diet I am among people & 29 & 46 & 15 & 4 & 6 \\
I adhere to diet when my general condition is good & 39 & 40 & 11 & 4 & 6 \\
\hline
\end{tabular}

Table 5. Total mean and standard deviation of patients with hypertension

\begin{tabular}{lllll}
\hline & mean & SD & MIN & MAX \\
\hline Sum of knowledge scores & 4.73 & 1.55 & 0 & 9 \\
Sum of perception scores & 29.58 & 3.94 & 22 & 40 \\
Sum of follow-up scores & 9.45 & 2.27 & 5 & 17 \\
\hline
\end{tabular}

\section{Discussion}

The results of present study conducted to evaluate the status of nutritional knowledge and disease perception and diet follow-up in patients with hypertension showed that those who have higher knowledge and perception of their disease are more adhere to their diet and are less suffer from its complications. In addition, people who believe their health status can be controlled are more responsible for their health and adhere to their diet. In the searches conducted by the researcher, few studies have been conducted on the understanding of the disease in people with hypertension in Iran, which shows the need for more research in this field. The researcher found a few studies conducted on the perception of the disease in people with hypertension in Iran, indicating the need to conduct more studies in this area. Results of this study are in line with the study conducted by Booth et al (2014), who recommended enhancing the knowledge of patients in this regard.

The results of their study showed that education and follow-up care resulted in a significant decrease in smoking and reduced salt intake, led to reduction in recurrence of heart disease and mortality in patients (Booth III et al., 2014). Providing education for patients could prevent the complications of the disease. The results of this study can be used to inform health service managers and service staff to make the necessary actions to increase the follow-up trainings. Adherence to the diet in patients with hypertension is very important. This important factor reduces the frequent visits and admissions to emergencies and clinics, which are psychologically and economically problematic for the patient and family. High and uncontrolled blood pressure, in addition to imposing the cost of hospitalization, is associated with recurrent disease, reduced independent performance of patient, reduced quality, feeling disability, lack of self-confidence in the patient. In line with these studies, Babaei Siset al. (2016) conducted a study entitled "The effect of educational intervention on lifestyle modification on blood pressure control in patients with high blood pressure." This research was a quasi-experimental study. Based on the results, after the intervention, the mean scores of variables of doing exercise, weight control, nutrition and mental health in the experimental group were significantly increased $(\mathrm{P}<0.05)$, but the changes in the control group were not significant $(0.05<\mathrm{P})$. Educational intervention had a significant effect on the reduction of blood pressure in the experimental group compared to the control group $(\mathrm{P}<0.05)$. The findings of the study showed that educational intervention was effective in enhancing the physical activity, improving weight control and nutrition, and improving mental health in patients with hypertension (Babaei-Sis et al., 2016). The results of the study indicate that the nutritional knowledge of patients should be increased.

- Other studies consistent with the present study included the studies conducted by Cheraghi et al. (2015) and Hosseini et al. (2014). It can be stated that y enhancing the knowledge about the diet therapy complications of the disease can be reduced. The researcher found a few studies conducted on the perception of the disease in people with hypertension in Iran, indicating the need to conduct more studies in this area. In a study conducted by Kamran et al. (2015), perception was considered as a determining factor in the diet of patients with hypertension (Kamran, Sharifirad, \& Mohebi, 2013). Perception has been considered as an important factor in nutritional behavior in the studies conducted in this regard. The results are also justifiable with behavioral theories, since planning for a behavior depends on the outcomes and benefits predicted. Rajpura and Nayak in their study reported a positive relationship between perception of the disease and positive beliefs about treatment in the elderly people with hypertension adhering the treatment (Rajpura \& Nayak, 2014). Moreover, the results of the study conducted by Kang et al. (2015) showed that patients who have higher perception of their disease are more adhering to their diet 
(Hacihasanoğlu \& Gözüm, 2011). The results of the study conducted by Saarti et al showed that the mean scores of disease perception in patients who followed good treatment were higher than those who had weaker adherence to treatment, but the relationship was not significant (Saarti et al., 2016).

In this study, it was found that home care and training improved the perception of diet and its association with the disease in people with hypertension was confirmed. Thus, this type of training can be an important step in enhancing the patients' perception of their status, and subsequently, improving adherence to special therapeutic and food diet. In the area of adherence to diet, results show that diet follow-up can play an important role in improving hypertension and according to the results found in the present study; the need to follow a diet is completely being felt. In line with result of this study, Ali Mohammadi et al. (2006) conducted a study to examine the effect of follow-up on blood pressure control in patients with hypertension. Based on the results, follow-up improved the diet of patients with hypertension. With regard to the nutritional model, significant difference was found between the two groups after the intervention in accepting the diet by patients.

Results of studies conducted by Rahimi et al. (2006) or Hacihasanoğlu et al. (2011), are in line with results of this study on the need to follow-up the diet. According to the results of this study, it can be concluded that by implementing a follow-up care model, there is a significant difference in the level of general and specific dimensions of patients' quality of life, so that the implementation of this care model is associated with an increase in the quality of life of patients.

-In line with this study, a study was conducted by Hashemi et al. (2014) entitled "The impact of adherence to diet by hemodialysis patients". This study was a clinical trial study performed on 98 hemodialysis patients in two hospitals of Baqiyatallah and Chamran in 2013. Based on the results, the hemodialysis patients did not follow proper diet. Significant relationship was found between follow-up care and adherence to diet $(p=0.0001)$. Based on the study results, patients were affected by the follow-up care model through counseling and providing education on adherence to a better diet. Thus, it is recommended that nurses to use this model to prevent complications and improve the hemodialysis patients' diet (Hashemi, Tayebi, Rahimi, \& Einollahi, 2015).

The strength point of this study is the presence of a specific location for sampling, but due to inclusion criteria such as lack of underlying illness, a large number of samples were excluded from the study. Some limitations of present study were self-reporting nature of the questionnaires, which might be completed inaccurately, lack of access to experiments related to chemical and structural changes, and medical instruments.

\section{Conclusion}

The results of this study showed that is need to enhance the knowledge and perception and adherence to diet in patients with hypertension and non-adherence to drug and non-drug diet is associated with complications of hypertension, such as stroke. By studying the status of dietary knowledge and perception of the disease and increasing it and adherence to an an effective diet, the complications of the disease in people with hypertension can be reduced.

\section{Acknowledgement}

This paper was adopted from the master thesis on nursing and community health approved by the Research Council of Hamedan University of Medical Sciences and Health Services in 1396/02/01 The project was also approved and recorded at Ethics Committee with research number of IR.UMSHA.REC.1396.41 and at the Iran's Clinical Registry Center with code of IRCT201704149014N157.

Authors of this study thereby appreciate honorable research deputy and faculty members of Nursing Faculty of Hamedan University of Medical Sciences, honorable staff of Farshchian Cardiovascular Clinic, and patients participating in this research.

\section{References}

Babaei-Sis, M., Ranjbaran, S., Mahmoodi, H., Babazadeh, T., Moradi, F., \& Mirzaeian, K. (2016). The Effect of Educational Intervention of Life Style Modification on Blood Pressure Control in Patients with Hypertension. $J$ Educ Community Health, 3(1), 12-19.

Booth III, J. N., Levitan, E. B., Brown, T. M., Farkouh, M. E., Safford, M. M., \& Muntner, P. (2014). Effect of sustaining lifestyle modifications (nonsmoking, weight reduction, physical activity, and mediterranean diet) after healing of myocardial infarction, percutaneous intervention, or coronary bypass (from the REasons for Geographic and Racial Differences in Stroke Study). The American journal of cardiology, 113(12), 19331940. 
Cheraghi, F., Mortazavi, S. Z., Shamsaei, F., \& Moghimbeigi, A. (2014). Effect of education on management of blood glucose in children with diabetes. Journal of Nursing Education, 3(1), 1-11.

Hacihasanoğlu, R., \& Gözüm, S. (2011). The effect of patient education and home monitoring on medication compliance, hypertension management, healthy lifestyle behaviours and BMI in a primary health care setting. Journal of clinical nursing, 20(5-6), 692-705.

Hashemi, S., Tayebi, A., Rahimi, A., \& Einollahi, B. (2015). Examining the effect of continuous care model on adherence to dietary regimen among patients receiving hemodialysis. Iran J Crit Care Nurs, 7(4), 215-20.

Hosseini, F., Farshidi, H., Aghamolaei, T., Madani, A., \& Ghanbarnejad, A. (2014). The impact of an educational intervention based on PRECEDE-PROCEED model on lifestyle changes among hypertension patients. Iranian Journal of Health Education and Health Promotion, 2(1), 17-27.

Kamran, A., Sharifirad, G., \& Mohebi, S. (2013). Psychometric assessment of nutritional knowledge, illness perceptions and dietary adherence in hypertensive patients-Ardabil. Health Systems Research, 1774-1785.

Kang, C. D., Tsang, P. P., Li, W. T., Wang, H. H., Liu, K. Q., \& Griffiths, S. M. (2015). Determinants of medication adherence and blood pressure control among hypertensive patients in Hong Kong: a cross-sectional study. International journal of cardiology, 182, 250-7.

Linderholm, M., \& Friedrichsen, M. (2010). A desire to be seen: Family caregivers' experiences of their caring role in palliative home care. Cancer nursing, 33(1), 28-36.

Mills, K. T., Bundy, J. D., Kelly, T. N., Reed, J. E., Kearney, P. M., Reynolds, K., ... \& He, J. (2016). Global Disparities of Hypertension Prevalence and ControlClinical Perspective: A Systematic Analysis of Population-Based Studies From 90 Countries. Circulation, 134(6), 441-450.

Mohammadi, M. A., Dadkhah, B., Sazavar, H., \& Mozaffari, N. (2006). The effect of follow up on blood pressure control in hypertensive patients. Journal of Ardabil University of Medical Sciences, 6(2), 156-162.

Mohebi, S., Azadbakhat, L., Feyzi, A., Hozoori, M., Kamran, A., \& Sharifirad, G. (2013). Educational Needs of Women with Metabolic Syndrome on Healthy Nutrition in Isfahan: Application of Health Promotion Model. journal of health, 4(2), 165-179.

Ndumele, C. D., Shaykevich, S., Williams, D., \& Hicks, L. S. (2010). Disparities in adherence to hypertensive care in urban ambulatory settings. Journal of health care for the poor and underserved, 21(1), 132-143.

Rahimi, A., Ahmadi, F., \& Gholyaf, M. (2006). Effects of Applying Continuous Care Model on Quality of Life in Hemodialysis Patients. Razi Journal of Medical Sciences, 13(52), 123-34.

Rajpura, J. R., \& Nayak, R. (2014). Role of illness perceptions and medication beliefs on medication compliance of elderly hypertensive cohorts. Journal of pharmacy practice, 27(1), 19-24.

Saarti, S., Hajj, A., Karam, L., Jabbour, H., Sarkis, A., El Osta, N., \& Khabbaz, L. R. (2016). Association between adherence, treatment satisfaction and illness perception in hypertensive patients. Journal of human hypertension, 30(5), 341.

Sanne, S., Muntner, P., Kawasaki, L., Hyre, A., \& DeSalvo, K. B. (2008). Hypertension knowledge among patients from an urban clinic. Ethnicity and Disease, 18(1), 42.

Sheahan, S. L., \& Fields, B. (2008). Sodium dietary restriction, knowledge, beliefs, and decision-making behavior of older females. Journal of the American Academy of Nurse Practitioners, 20(4), 217-224.

\section{Copyrights}

Copyright for this article is retained by the author(s), with first publication rights granted to the journal.

This is an open-access article distributed under the terms and conditions of the Creative Commons Attribution license (http://creativecommons.org/licenses/by/4.0/). 